\title{
Dipole Moments and Molecular Association of Some Picrates of Primary, Secondary, and Tertiary Amines in Benzene and Dioxane
}

\author{
By Arthur A. Maryott
}

\begin{abstract}
The dielectric constants of dilute benzene and dioxane solutions of primary, secondary, and tertiary amine salts of picric acid were measured. Dipole moments are reported for the picrates of the following amines; in benzene: tri-iso-amyl- (11.9), tri- $n$-butyl- (11.9), triethyl (11.7), diethylbenzyl- (11.8), di- $n$-butyl- (11.5), di- $n$-propyl- (11.5); in dioxane: tri- $n$-butyl(12.2), di- $n$-butyl- (12.1), $n$-octyl- (12.1), and $n$-octadecyl- (12.3). The picrates of primary and secondary amines show a pronounced tendency to associate in benzene, as evidenced by the nonlinear variation in dielectric constant with concentration, but not in dioxane. The effect of the size and shape of the alkylammonium ion and of hydrogen bonding on the association are discussed.
\end{abstract}

\section{Introduction}

Electrolytes dissolved in nonpolar solvents exhibit behavior that is often complicated by intermolecular associations and interactions not encountered in the more familiar aqueous systems. The properties of certain types of organic salts have been investigated rather extensively in such solvents by Kraus [1] ${ }^{1}$ and coworkers. In brief, ionic dissociation occurs only to a very minute extent. The salt exists almost entirely as pairs of oppositely charged ions held together by coulombic forces. By comparison with ordinary molecules, these ion pairs have unusually large dipole moments, which frequently promote association into dimers or other higher aggregates. The extent of dipole association depends on a variety of physical factors such as the magnitude of the dipole moment, the size and shape of the molecules, and the dielectric constant of the medium.

Another important interaction is the formation of hydrogen bonds. Thus both physical and chemical properties of the solute and solvent determine the extent and nature of the association. These factors have a particular bearing on acidbase equilibria and indicator reactions [2] in

\footnotetext{
1 Figures in brackets indicate the literature references at the end of this paper.
}

media of low dielectric constant. Consequently, it is not surprising that these equilibria frequently do not follow a simple mass-law relationship.

The measurement of dielectric constant affords a convenient method of studying these interactions, and the dipole moments derived from these measurements are useful in interpreting and correlating the results. Although a wide variety of salts of organic acids and bases are soluble in media of low dielectric constant, previous studies have been confined largely to a few quaternary and tertiary ammonium salts. In this investigation, the dielectric constants were determined for dilute solutions of some primary, secondary, and tertiary amine salts of picric acid in benzene and in dioxane. The amines were all of sufficient strength to prevent measurable dissociation of the picrates into free acid and base.

\section{Equipment and Materials}

The equipment for measuring the dielectric constants, the procedure of making up the solutions directly in the cell, and the purification of the solvents were the same as described previously [3] except for the following changes. An oil bath replaced the air bath. A glass cell having cylindrical platinum electrodes and described 
elsewhere [4] was used in place of the silver filmglass cell for a part of the measurements. All measurements were made at $30^{\circ} \pm 0.01^{\circ} \mathrm{C}$.

The picrates were prepared by dissolving a given amount of recrystallized picric acid in hot ethanol or benzene and adding slightly more than the required amount of amine. They were purified by one or more recrystallizations from alcohol or benzene and then dried to constant weight in a vacuum oven. The melting points were as follows: triisoamylammonium picrate, $126^{\circ} \mathrm{C}$; tri- $n$-butylammonium picrate, $106^{\circ} \mathrm{C}$; triethylammonium picrate, $173^{\circ} \mathrm{C}$; diethylbenzylammonium picrate, $121^{\circ} \mathrm{C}$; di- $n$-butylammonium picrate, $63^{\circ} \mathrm{C}$; di- $n$-propylammonium picrate, $97^{\circ} \mathrm{C}$; n-octylammonium picrate, $112^{\circ} \mathrm{C}$; and $n$-octadecylammonium picrate, $108^{\circ} \mathrm{C}$.

\section{Experimental Results}

The experimental data are ineluded in table 1. The second, third, and last columns give, respectively, the concentration of solute, $N_{2}$, in mole fraction, the difference in dielectric constant between the solution and the solvent, $\Delta \epsilon$, and the quotient, $\Delta \epsilon / N_{2}$.

TABLE 1. Experimental data for substituted ammonium picrates in benzene and dioxane

\begin{tabular}{c|c|c|c}
\hline \hline Picrate of- & $N_{2} \times 10^{4}$ & $\Delta \epsilon$ & $\Delta \epsilon / N_{2}$ \\
\hline
\end{tabular}

IN DIOXANE

\begin{tabular}{|c|c|c|c|}
\hline \multirow{6}{*}{ Tri- $n$-butylamine... } & $(0.0000$ & & 204 \\
\hline & .4004 & 0.0081 & 202 \\
\hline & .8880 & .0181 & 204 \\
\hline & 1. 792 & .0366 & 204 \\
\hline & 2. 697 & .0553 & 205 \\
\hline & 3.490 & .0717 & 205 \\
\hline \multirow{6}{*}{ Di- $n$-butylamine } & $(0.0000$ & & 202 \\
\hline & .4838 & .0097 & 201 \\
\hline & 1. 302 & .0263 & 202 \\
\hline & 2. 340 & .0475 & 203 \\
\hline & 3.416 & .0693 & 203 \\
\hline & 0.0000 & 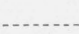 & 207 \\
\hline \multirow{5}{*}{ n-Octadecylamine } & .4212 & .0087 & 207 \\
\hline & 1.013 & .0210 & 207 \\
\hline & 1. 943 & .0398 & 205 \\
\hline & 2. 977 & .0599 & 201 \\
\hline & 3.953 & .0789 & 200 \\
\hline \multirow{6}{*}{ n-Octylamine.. } & 0.0000 & & 201 \\
\hline & .4572 & .0092 & 201 \\
\hline & 1. 242 & .0250 & 201 \\
\hline & 2. 149 & .0430 & 200 \\
\hline & 3.130 & .0618 & 198 \\
\hline & 3.950 & .0774 & 196 \\
\hline
\end{tabular}

TABLE 1. Experimental data for substituted ammonium picrates in benzene and dioxane-Continued



Considering first the data in dioxane, it is evident that the picrates of primary, secondary, and tertiary amines behave similarly. The dielectric constant is substantially a linear function of the concentration as indicated by the constancy of the ratio, $\Delta \epsilon / N_{2}$. Such a linear relationship appears to be general and characteristic of dilute solutions in cases where the molecular state of the solute is unaffected by changes in concentration. 
It is taken here as the criterion indicating lack of association of the solute. ${ }^{2}$

In benzene only the picrates of tertiary amines show a linear variation of dielectric constant with concentration and, consequently, the absence of association. A representative comparison of the data in benzene with that in dioxane is shown in figure 1 where $\Delta \boldsymbol{\epsilon} / N_{2}$ is plotted as a function of $N_{2}$ for tributyl-, dibutyl-, and octylammonium picrate. The data for octylammonium picrate in

\footnotetext{
2 The usual criterion involving constancy of the molar polarization calculated from the Clausius-Mossotti equation is less acceptable experimentally. If the dielectric constant varies linearly with the concentration, the molar polarization cannot be strictly independent of the concentration, although the change is small for small changes in dielectric constant. This distinction is of some consequence in the present case, as the dipole moments are sufficiently large to cause a substantial change in dielectric constant even at quite low concentrations.

In special cases, association might occur without altering the linear relation between dielectric constant and concentration. This would be true, for example, if two molecules associated with their dipoles at right angles or else with equal probability of parallel and antiparallel alinement. This interpretation, however, can hardly apply to the present case where the shape of the molecules is such as to favor an antiparallel alinement.
}

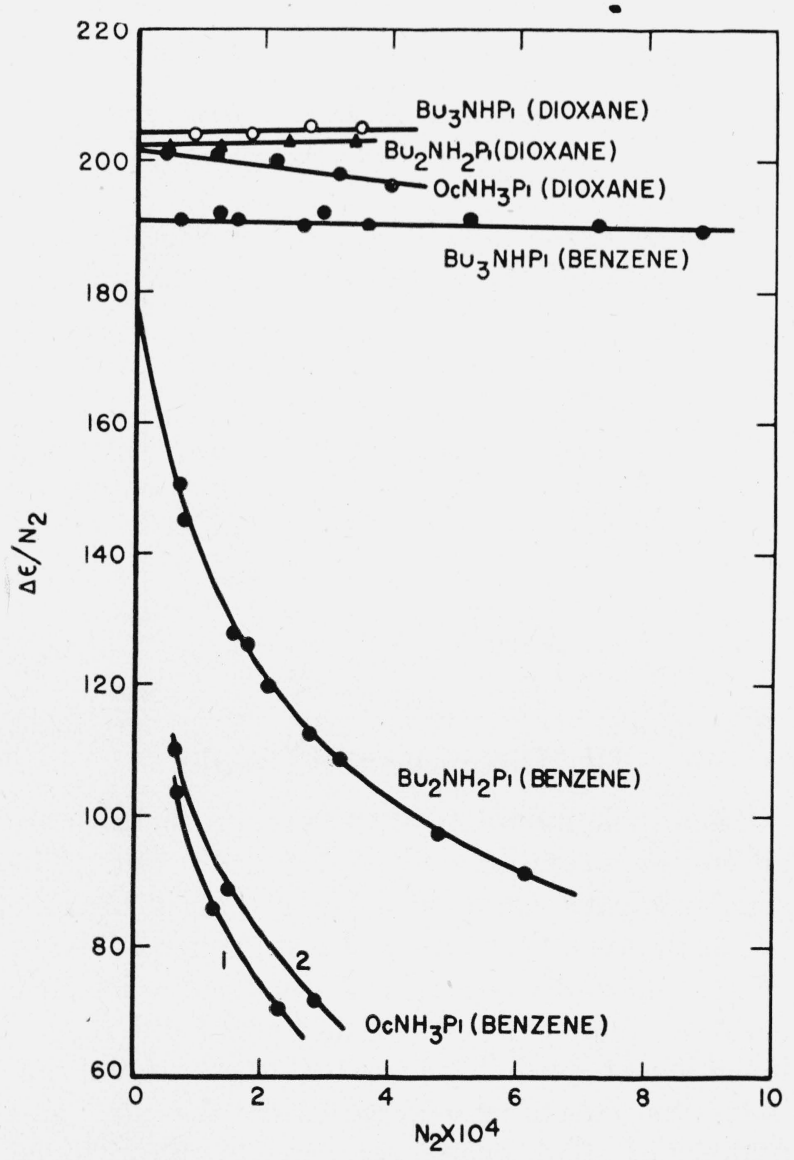

FIGURE 1. Representative data for the picrates of primary, secondary, and tertiary amines in benzene and in dioxane. benzene are not included in table 1 , because the solubility was not sufficient to permit accurate measurements by the procedure used in the remaining cases. These measurements were obtained by adding successive amounts of a solution of picric acid to the cell containing solvent to which sufficient octylamine had been added. The observed changes in dielectric constant then require a small correction to compensate for changes in concentration of the amine. By this method concentrations up to the solubility limit of the picrate could be measured. Although this indirect procedure was checked for tributylammonium picrate and gave agreement with the direct measurements, the results for octylammonium picrate depended on the amount of base initially present. The curve for measurement 2 , in which the initial concentration of base was greater than for measurement 1 , falls above the latter curve. In this case it would appear that the presence of free amine interferes with the association of the picrate and that the limiting curve for the salt alone would fall below either of the experimental curves.

The molar polarization at infinite dilution, $P_{2 \infty}$, the electronic polarization, $P_{E}$, and the dipole moment, $\mu$, for each picrate are given in table 2. $\quad P_{2 \infty}$ was calculated from the Hedestrand

TABle 2. Molar polarization at infinite dilution, $P_{2_{\infty}}$, electronic polarization, $P_{E}$, and dipole moment, $\mu$, for the various picrates

\begin{tabular}{|c|c|c|c|c|}
\hline Picrate of- & Solvent & $P_{2 \infty}$ & $P E$ & $\mu \times 10^{18} \mathrm{esu}$ \\
\hline Tri-i-amylamine & Benzene & 2,950 & 120 & 11.9 \\
\hline Tri- $n$-butylamine & $\ldots$ do $\ldots \ldots$ & 2,940 & 106 & 11.9 \\
\hline Do & Dioxane & 3,090 & 106 & 12. 2 \\
\hline Triethylamine & Benzene..... & 2,850 & 78 & 11.7 \\
\hline Diethylbenzylamine ...... & $\ldots$. & 2,910 & 98 & 11.8 \\
\hline Di-n-butylamine & $\ldots$. do & 2,730 & 88 & 11.5 \\
\hline Do & Dioxane & 3,040 & 88 & 12.1 \\
\hline Di- $n$-propylamine & Benzene....- & 2,750 & 78 & 11.5 \\
\hline$n$-Octadecylamine & Dioxane & 3,150 & 133 & 12.3 \\
\hline$n$-Octylamine & $\ldots$ do _..._. & 3,030 & 88 & 12.1 \\
\hline
\end{tabular}

equation [5], using the values of $\Delta \epsilon / N_{2}$ shown opposite zero concentration in table $1 .^{3}$ These values for zero concentration represent averages

\footnotetext{
${ }^{3}$ In these calculations the densities of all the benzene solutions were assumed to be $d_{801}=d_{\text {benzenet }}+1.1 \quad N_{2}$ and of the dioxane solutions, $d_{801}=$ $d_{\text {dioxane }}+0.5 N_{2}$. These relations are averages based partly on some experimental data and partly on calculations involving the densities of the molten salts [6] and the assumption of additivity of molar volumes. They are adequate for the present purpose, inasmuch as complete neglect of any changes in density would not affect the moments calculated by more than 1 percent in any case.
} 
for those cases where there was no significant drift in values with concentration and extrapolated values in the remaining cases. $P_{E}$ was taken as the sum of the atomic refractivities listed in Landolt-Börnstein Tabellen, fifth edition. The dipole moment was calculated from the equation,

$$
\mu=0.1281\left[\left(P_{2 \infty}-P_{E}\right) T\right]^{1 / 2} \times 10^{-18} \mathrm{esu},
$$

where $T$ is the temperature in degrees Kelvin.

The dipole moments of two of these salts in benzene have been measured previously. The moment reported here for tributylammonium picrate is in agreement with that of Deitz and Fuoss [7], but the values reported by Geddes and Kraus [8] and by Mortier [9] for tributyl- and triamylammonium picrates are about 10 percent higher. The higher values probably can be attributed to small experimental errors in measuring the dielectric constant of the solvent or of the more dilute solutions. Such errors have a disproportionately large effect on the polarization at infinite dilution when obtained by the conventional extrapolation [10].

As the secondary ammonium picrates are associated in benzene and show a pronounced decrease in the value of $\Delta \epsilon / N_{2}$ with increasing concentration, the method of obtaining the polarization and dipole moment of the monomeric form needs explaining. A graphical extrapolation to get the limiting value $\Delta \epsilon / N_{2}$ is obviously subject to considerable uncertainty. The procedure adopted was essentially the same as employed by Pohl, Hobbs, and Gross in a study of the association of carboxylic acids [11]. It is reasonable to assume that, at the low concentrations involved, the formation of complexes higher than double molecules is not significant. The equilibrium constant for this dimermonomer equilibrium, $A_{2}=2 A$, may then be obtained in the following manner. The change in dielectric constant on addition of the solute of stoichiometric concentration, $N_{2}$, can be considered the sum of two independent contributions, one proportional to the concentration of monomer and the other proportional to the concentration of dimer.

Then

$$
\Delta \epsilon=\Delta \epsilon_{m}+\Delta \epsilon_{d}=k_{m} \alpha N_{2}+k_{d}(1-\alpha) N_{2}
$$

or

$$
\alpha=\left(\Delta \epsilon / N_{2}-k_{d}\right) /\left(k_{m}-k_{d}\right),
$$

where $\alpha$ is the fraction of solute that is in the monomeric form and $k_{m}$ and $k_{d}$ are the proportionality constants for the monomer and dimer, respectively. The equilibrium constant is then

$$
K_{N}=\frac{\alpha^{2} N_{2}}{\frac{1-\alpha}{2}}=\frac{\left(\Delta \epsilon / N_{2}-k_{d}\right)^{2} 2 N_{2}}{\left(k_{n t}-\Delta \epsilon / N_{2}\right)\left(k_{m}-k_{d}\right)} .
$$

The values of $k_{m}$ and $k_{d}$, adjusted by trial and error until they best fitted the experimental data in accordance with eq 3 , are listed in table 3 , together with the value of $K_{N}$ at each concentration. The numerical values of the equilibrium constants should be regarded as rather approximate because of the limited range of concentration studied, the assumption that no higher complexes are formed, and the sensitiveness of the constants to the exact values of $k_{m}$ and $k_{d}$. From $k_{m}$, which represents the value of $\Delta \epsilon / N_{2}$ for the monomeric form and can be determined quite critically, the polarization and

\begin{tabular}{|c|c|c|c|}
\hline \multicolumn{2}{|c|}{$\begin{array}{l}\text { Di- } n \text {-propylammonium } \\
\text { picrate }\end{array}$} & \multicolumn{2}{|c|}{$\begin{array}{l}\text { Di- } n \text {-butylammonium } \\
\text { picrate }\end{array}$} \\
\hline$N_{2} \times 10^{4}$ & $K_{N} \times 10^{4}$ & $N_{2} \times 10^{4}$ & $K_{N} \times 10^{4}$ \\
\hline 0. 3892 & 7. 2 & 0.7044 & 5.8 \\
\hline .4466 & 7. 7 & .7660 & 4.8 \\
\hline 1. 189 & 8.0 & 1.525 & 4.8 \\
\hline 1. 665 & 7. 7 & 1. 784 & 5. 2 \\
\hline 2. 022 & 7.8 & 2.090 & 4. 8 \\
\hline 2. 839 & 7. 6 & 2. 740 & 4. 9 \\
\hline 3. 069 & 7.8 & 3. 232 & 5.0 \\
\hline 3. 921 & 7.8 & 4. 782 & 4.8 \\
\hline 4. 075 & 7.6 & 6.133 & 5.0 \\
\hline 4. 873 & 7.8 & & \\
\hline \multicolumn{2}{|c|}{$\begin{aligned} k_{m} & =179 \\
k_{d} & =15\end{aligned}$} & \multicolumn{2}{|c|}{$\begin{aligned} k_{m} & =178 \\
k_{d} & =15\end{aligned}$} \\
\hline
\end{tabular}
dipole moment are obtained.

TABLE 3.-Dimer-monomer equilibrium constants, $K_{N}$, in benzene

\section{Discussion of Results}

The dipole moments of the various picrates are all in the neighborhood of $12 \times 10^{-18}$ esu. The slightly higher values in dioxane than in benzene are in the direction expected from general solvent effects, since dioxane has a slightly lower dielectric constant than benzene. Other small differences, if significant, probably are attributable to varying inductive effects associated with the size and number of alkyl groups attached to the nitrogen atom. There is no evidence of any steric effect from these alkyl groups that would influence the 
distance of approach of the two ions that make up the ion pair. The negatively charged phenolate oxygen evidently comes into contact with the alkylammonium ion on the side occupied by a hydrogen atom. The distance of separation of the charges on the two ions is about $2.5 \mathrm{~A}$ in all cases.

It was noted in the preceding section that picrates of the primary and secondary amines show substantial association in benzene, whereas those of the tertiary amines do not. As the dipole moments are practically the same, the explanation is evidently related to differences in the size and shape of the alkylammonium ion. In the case of the tertiary ammonium picrates, the presence of three relatively large alkyl groups prevents sufficiently close approach of neighboring ion pairs to lead to association. Replacement of one or two of these alkyl groups by the much smaller hydrogen atoms, as in the secondary and primary ammonium picrates, enables the ion pairs to come close enough together to form stable dimers. As these picrates may be regarded as roughly ellipsoidal in shape with the dipole near the center and directed along the long axis, the most stable arrangement of the dimer would be with the two dipoles side by side and antiparallel. Other configurations in which the dipoles are inclined at an angle somewhat less than $180^{\circ}$ are also probable, so that the effective moment would not necessarily be zero. According to the value of $k_{d}$ listed in table 3 for dibutyl- and dipropylammonium picrate, the effective moment of the dimer is in the neighborhood of $5 \times 10^{-18}$ esu.

By employing the dipole association theory of Fuoss [12] ${ }^{4}$, an estimate of the distance of closest approach of ion pairs can be made from the initial slope of the polarization-concentration curve. This slope can be obtained for the two secondary ammonium picrates by combining eq 3 with the Hedestrand equation and differentiating. The distance of closest approach is calculated to be $4.5 \mathrm{~A}$ for dibutyl-ammonium picrate and $4.6 \mathrm{~A}$ for dipropylammonium picrate. An estimate only of the lower limit of this distance is possible for the tertiary ammonium picrates where no

\footnotetext{
4 The theory assumes an ellipsoidal molecular model with a point dipole at the center directed along the major axis. The present calculations assume that the ratio of the minor to major axes is $1: 2$.
}

significant association is indicated. A value of $5.7 \mathrm{~A}$ is calculated on the basis that not more than 1 percent is associated at $5 \times 10^{-4}$ mole fraction. These dimensions are of reasonable magnitudes and indicate that dipole interactions alone are sufficient to account for differences in behavior of the picrates in benzene.

In dioxane none of the picrates shows any pronounced tendency to associate, although the small decrease in the values of $\Delta \epsilon / N_{2}$ with increasing concentration for the two primary ammonium picrates suggests a very slight association. There is evidently some interaction between the dioxane and the primary and secondary ammonium picrates that prevents as close an approach of ion pairs in this solvent as in benzene. In the secondary ammonium picrates, one of the two ammonium hydrogens can form a hydrogen bond with a dioxane molecule. The second of these hydrogens is evidently held up between the electrostatic bond and does not interact with the solvent; otherwise the dipole moment would be larger than observed. In the picrates of primary amines, the alkylammonium ion can interact with two molecules of dioxane so that again the effective size of the ion is sufficient to minimize the association of ion pairs. The effect of octylamine in diminishing the association of octylammonium picrate in benzene can be interpreted in the same manner. In this case, the amine acts as the acceptor molecule in the formation of hydrogen bonds.

These results are in accord with the conclusions of Davis and Schuhmann [2] of this laboratory based on spectrophotometric studies with indicators and show that the formation of hydrogen bonds and dipole association are important factors to be considered in interpreting acid-base behavior in media of low dielectric constant. By the proper choice of systems, it should be possible to realize simple mass-law relationships. In general, one would expect reactions of tertiary amines with organic acids to behave more nearly normal than those of primary and secondary amines in an inert solvent like benzene, whereas use of a protonacceptor solvent such as dioxane should tend to normalize the reactions of primary and secondary amines. 


\section{References}

[1] C. A. Kraus, J. Franklin Inst., 225, 687 (1938).

[2] M. M. Davis and P. J. Schuhmann, J. Research NBS 39, 221 (1947) RP1825.

[3] A. A. Maryott and S. F. Acree, J. Research NBS 38, 505 (1947) RP1791.

[4] A. A. Maryott, J. Research NBS 38, 527 (1947). RP1794.

[5] G. Hedestrand, Z. physik. Chem. [B] 2, 428 (1929).

[6] P. Walden, H. Ulich, and E. J. Bin, Z. physik. Chem. [A] 130, 495 (1927).
[7] V. Deitz and R. M. Fuoss, J. Am. Chem. Soc. 60, 2394 (1938).

[8] J. A. Geddes and C. A. Kraus, Trans. Faraday Soc. 32, 585 (1936).

[9] P. Mortier, Wis. Natuurkund. Tijdschr. 10, 114 (1941).

[10] I. F. Haverstadt and W. D. Kumler, J. Am. Chem. Soc. 64, 2988 (1942).

[11] H. A. Pohl, M. E. Hobbs, and P. M. Gross, J. Chem. Phys. 9, 408 (1941).

[12] R. M. Fuoss, J. Am. Chem. Soc. 56, 1031 (1934).

Washington, April 16, 1948. 